\title{
NORMAL SUBGROUPS OF GROUPS WHICH ARE PRODUCTS OF TWO ABELIAN SUBGROUPS
}

\author{
LARRY E. KNOP 1
}

\begin{abstract}
It is shown that if a group $G=A B$, where $A$ and $B$ are Abelian subgroups of $G, A \neq B$, and either $A$ or $B$ satisfies the maximum condition, then there is a normal subgroup $N$ of $G$, $N \neq G$, such that $N$ contains either $A$ or $B$.
\end{abstract}

Summary and background. The purpose of this paper is to show that, if a group $G$ is a nontrivial product of two Abelian subgroups $A$ and $B$, and if either $A$ or $B$ satisfies the maximum condition, then there is a proper normal subgroup of $G$ containing either $A$ or $B$. (L. E. Knop [7]).

The basic impetus for this research is found in a paper of N. Itô [1]. In this paper, Itô showed that if $G$ is a product of two Abelian subgroups $A$ and $B$, then $G$ is metabelian. Furthermore, he showed that if $G$ is finite, then there is a normal subgroup of $G$, not the identity, which is contained in either $A$ or $B$, and also that there is a proper normal subgroup of $G$ which contains either $A$ or $B$.

Additional work on the problem of when there is a normal subgroup contained in one of the two factors has been done by P. M. Cohn [2], who extended Itô's result to the case where $A$ and $B$ are infinite cyclic, and N. F. Sesekin [3], who extended the result to the case where $A$ and $B$ are finitely connected. E. Schenkman [4] claimed that both of Itô's results could be extended to the case where both $A$ and $B$ satisfied the maximum condition. Unfortunately there was a flaw in the proof that either $A$ or $B$ contained a normal subgroup of $G$ (discovered by both Schenkman, Zentralblatt review [4], and W. R. Scott [5]), and the proof that either $A$ or $B$ was contained in a proper normal subgroup of $G$ was based on this result. B. Amberg and W. R. Scott [6, Theorem 4] did show that if either $A$ or $B$

Presented to the Society, January 20, 1972 under the title Groups which are products of two Abelian subgroups; received by the editors November 22, 1972.

AMS (MOS) subject classifications (1970). Primary 20E15, $20 \mathrm{~F} 30$.

Key words and phrases. Abelian subgroup, commutator subgroup, maximum condition on subgroups, metabelian groups, product of subgroups, torsion free rank, torsion subgroup.

${ }^{1}$ This paper is part of the author's doctoral dissertation written under the direction of Professor W. R. Scott at the University of Utah. 
satisfies the maximum condition and if any one of three other conditions is satisfied, or, if both $A$ and $B$ are cyclic, then there is a proper normal subgroup containing one of the factors. This paper shows that additional conditions are not necessary, and establishes half of Schenkman's claim in a more general setting.

It should also be noted that recently B. Amberg [8] has announced that both of Schenkman's results hold in the more general setting where only one of the two factors is assumed to satisfy the maximum condition, and that additional facts about the structure of $G=A B$ where both $A$ and $B$ are Abelian subgroups satisfying the maximum condition can be established.

Notation, definitions, and lemmas. $\langle H\rangle$ is the subgroup generated by the set $H .(g, h)=g^{-1} h^{-1} g h . G^{\prime}=\langle(g, h)| g$ and $h$ are in $\left.G\right\rangle$ is the commutator subgroup of $G$. $G$ is metabelian provided $G^{\prime}$ is Abelian. $C(D)=\{x \mid x d=d x$ for all $d \in D\}$ is the centralizer in $G$ of the set $D . Z=C(G)$ is the center of $G$.

A group $A$ satisfies the maximum condition provided any ascending chain of subgroups is finite. For Abelian groups, this is equivalent to the condition that $A$ is finitely generated.

For a finitely generated Abelian group $A$, let $R(A)$ be the torsion free rank of $A$ and let $T(A)$ be the order of the torsion subgroup of $A$.

Let $G=A B$ be a product of Abelian subgroups and let $N$ be a normal subgroup of $G$. Define $A[N]=\{a \in A \mid a b \in N$ for some $b \in B\}$ and $B[N]=$ $\{b \in B \mid a b \in N$ for some $a \in A\}$.

The following elementary result will be used often enough to deserve explicit mention.

Lemma 1. If $C, D$, and $K$ are subgroups of the group $H=C D$, and $C \subseteq K$, then $K=C(D \cap K)$.

The following result of Itô is, of course, basic to this work.

LemMa 2 (Itô [1, Theorem 1]). Each ( finite or infinite) group, which may be represented as the product of two Abelian subgroups, is metabelian.

The next result can essentially be found in both Sesekin [3] and Schenk$\operatorname{man}[4]$.

Lemma 3. If $G=A B$ is a product of Abelian sugbroups, and $N$ is a normal subgroup of $G$, then $N A[N]=N B[N]=A[N] B[N]$ is a normal subgroup of $G$.

Proof. $A[N]=A \cap N B$ so $A[N]$ is a subgroup. $N$ is normal in $G$ so $N A[N]$ is also a subgroup. For any $a_{0} \in A[N], b \in B[N]$, there is an element 
$a \in A[N]$ such that $a b \in N$. Thus $a_{0} b=\left(a_{0} a^{-1}\right)(a b) \in A[N] N$. On the other hand, $N \subseteq A[N] B[N]$ by definition. Thus $N A[N]=A[N] B[N]$. Similarly, $N B[N]=A[N] B[N]$.

$\boldsymbol{A}$ is Abelian so $\boldsymbol{A}$ normalizes $N A[N]$. $B$ normalizes $N B[N]$. Thus $A[N] B[N]$ is normal in $G$.

Finally this last lemma, which can be found in Schenkman [4], will turn out to be crucial.

LEMma 4. If $H=\langle a\rangle C$, where $C$ is a normal Abelian subgroup and $a$ is an element of $H$, then $H^{\prime}=\{(a, c) \mid c \in C\}$.

Proof. Let $T=\{(a, c) \mid c \in C\}$. Then for any $c, c_{0} \in C,(a, c)\left(a, c_{0}\right)=$ $a^{-1} c^{-1} a c\left(a^{-1} c_{0}^{-1} a\right) c_{0}=a^{-1} c^{-1} a a^{-1} c_{0}^{-1} a c c_{0}=\left(a, c c_{0}\right)$ since $C$ is normal and Abelian. Also $(a, c)^{-1}=c^{-1} a^{-1} c a=a^{-1} c a c^{-1}=\left(a, c^{-1}\right)$ for the same reasons. Thus $T$ is a subgroup.

Now $H / C$ is Abelian, so we have $T \subseteq H^{\prime} \subseteq C$. Thus to show that $T$ is normal in $H$ it suffices to show that $T$ is invariant under conjugation by $a$. But $(a, c)^{a}=\left(a^{a}, c^{a}\right)=\left(a, c^{a}\right)$, so $T$ is normal in $H$.

Clearly $\langle a\rangle T / T$ is in the center of $H / T$. But since $C$ is Abelian, this means that $H / T$ is Abelian and $H^{\prime} \subseteq T$.

THEOREM. If the group $G=A B$, where $A$ and $B$ are Abelian subgroups of $G, A \neq B$, and either $A$ or $B$ satisfies the maximum condition, then there is $a$ normal subgroup $N$ of $G, N \neq G$, such that $A \subseteq N$ or $B \subseteq N$.

Proof. Assume $A$ satisfies the maximum condition.

Suppose the theorem is false. Let $G=A B$ be a counterexample.

(1) $G^{\prime} \neq 1, A \neq G, B \neq G$, and $A G^{\prime}=G=B G^{\prime}$.

Proof. $A \neq B$, and so either $G \neq A$ or $G \neq B$. If $G^{\prime}=1$, then $G$ is Abelian and so one of the factors would be a proper normal subgroup of $G$. Thus $G^{\prime} \neq 1$. Since $A$ and $B$ are Abelian, and $G$ is not, we have $A \neq G$ and $B \neq G$. $A G^{\prime}$ and $B G^{\prime}$ are normal subgroups of $G$ containing $A$ and $B$ respectively, so we must have $A G^{\prime}=G=B G^{\prime}$.

Now let $G=A B$ be a minimal counterexample in the following sense: If $G_{0}=A_{0} B_{0}$ is any other counterexample, then $R(A) \leqq R\left(A_{0}\right)$, and if $R(A)=R\left(A_{0}\right)$, then $T(A) \leqq T\left(A_{0}\right)$. In addition:

(2) We may assume $B \cap G^{\prime}=1$.

Proof. Let $D=B \cap G^{\prime}$. Both $B$ and $G^{\prime}$ are Abelian, so $G=B G^{\prime} \subseteq C(D)$. Thus $D \subseteq Z$, and in particular, $D$ is normal in $G$.

Suppose $A D=B$. Then we have $G=A B=A A D=A D=B$, and this is impossible by (1). So $A D \neq B$.

We now have $G / D=(A D / D)(B / D)$ and $A D / D \neq B / D$. Suppose there is a normal subgroup $N / D$ of $G / D, N / D \neq G / D$, such that $N / D$ contains either $A D / D$ or $B / D$. Then $N$ is normal in $G, N \neq G$, and $N$ contains $A$ or $B$. This 
contradicts our assumption that the theorem was false for $G=A B$, and so $G / D=(A D / D)(B / D)$ must also be a counterexample.

Now $A D / D \cong A \mid A \cap D$. If $A \cap D \neq 1$, then either $R(A D / D)<R(A)$ or $R(A D / D)=R(A)$ and $T(A D / D)<T(A)$. In either case we have a smaller than minimal counterexample and this is impossible. So $A \cap D=1$, $A D / D \cong A$, and $G / D$ is also a minimal counterexample.

In $G / D$ we have $(G / D)^{\prime}=G^{\prime} \mid D$ since $D \subseteq G^{\prime}$, and so $(G / D)^{\prime} \cap(B / D)=1$. Hence, without loss of generality, we may assume $B \cap G^{\prime}=1$.

(3) If $N$ is a normal subgroup of $G$ and $A N \neq B N$, then $A \cap N=1$.

Proof. Suppose false. Then $A \cap N \neq 1$. But $A N / N=A / A \cap N$, and so either $R(A N / N)<R(A)$ or $R(A N / N)=R(A)$ and $T(A N / N)<T(A)$. In either case, $G / N=(A N / N)(B N / N)$ is a group satisfying the hypothesis of the theorem, which is smaller than a minimal counterexample. Thus there is a normal subgroup $U / N \neq G / N$ of $G / N$ containing either $A N / N$ or $B N / N$. But this means that $U$ is a nontrivial normal subgroup of $G$, containing either $A$ or $B$. Contradiction.

(4) $A \cap Z=1$.

Proof. Suppose $A Z=B Z$. Then $G=A B=A A Z=A Z$. Both $A$ and $Z$ centralize $A$. Thus $A$ is in the center of $G$ and so $G=Z$. This contradicts $G^{\prime} \neq 1$, so $A Z \neq B Z$. By (3), $A \cap Z=1$.

(5) $A \cap B=1$ and $A \cap G^{\prime}=1$.

Proof. $A$ and $B$ centralize $A \cap B$, so $A \cap B \subseteq A \cap Z=1$. $A$ and $G^{\prime}$ centralize $A \cap G^{\prime}$ since $G^{\prime}$ is Abelian by Lemma 2 , and since $G=A G^{\prime}, A \cap G^{\prime} \subseteq$ $A \cap Z=1$.

Now $A \neq 1$ since $G$ is not Abelian. Let $a \neq 1$ be an element of $A$. Let $G_{0}=\left(\langle a\rangle G^{\prime}\right)^{\prime} .\langle a\rangle G^{\prime}$ is normal in $G$ and $G_{0}$ is characteristic in $\langle a\rangle G^{\prime}$, so $G_{0}$ is normal in $G$. Clearly $G_{0}$ is a subgroup of $G^{\prime}$, and so $A \cap G_{0}=1=B \cap G_{0}$.

(6) $G_{0} \neq 1$.

Proof. Suppose $G_{0}=1$. Then $G^{\prime}$ centralizes $a$. $A$ is also in the centralizer of $a$, so $a \in A \cap Z$. But $a \neq 1$ and this contradicts (4).

Let $A_{0}=A\left[G_{0}\right]$ and $B_{0}=B\left[G_{0}\right]$. Let $\bar{G}=A_{0} B_{0}=A_{0} G_{0}=B_{0} G_{0}$. By Lemma $3, \bar{G}$ is normal in $G$.

(7) $\bar{G}=G$.

Proof. Suppose false. $G \neq G$. Then

$$
A \bar{G}=A A_{0} B_{0}=A B_{0} \text { and } B \bar{G}=\bar{G} B=A_{0} B_{0} B=A_{0} B .
$$

Suppose $A \bar{G}=B \bar{G}$. Then $A B_{0}=A_{0} B$. By Lemma $1, B=B_{0}(A \cap B)=B_{0}$. Thus $B$ is a subgroup of a proper normal subgroup $\bar{G}$, and this is impossible. Therefore $A \bar{G} \neq B \bar{G}$.

By (3), $A \cap \bar{G}=1$. Thus $A_{0}=1 . \bar{G}=A_{0} B_{0}$, so $\bar{G}=B_{0}$. But then $G_{0} \subseteq B$, and thus $G_{0}=1$ since $G_{0} \subseteq G^{\prime}$. This contradicts (6) however, so (7) holds.

(8) $A_{0}=A, B_{0}=B$, and $G_{0}=G^{\prime}$. 
Proof. $G=\bar{G}$, so $A B=A_{0} B_{0}$. By Lemma 1 ,

$$
A=A_{0}\left(A \cap B_{0}\right) \subseteq A_{0}(A \cap B)=A_{0} .
$$

Similarly $B=B_{0}$. We also have

$$
G=A G^{\prime}=A_{0} G_{0} \text {, so } G^{\prime}=G_{0}\left(A_{0} \cap G^{\prime}\right) \subseteq G_{0}\left(A \cap G^{\prime}\right)=G_{0},
$$

so $G^{\prime}=G_{0}$.

We can now conclude the proof. By Lemma 4, $G_{0}=\left\{(a, g) \mid g \in G^{\prime}\right\}$. But $a \in A=A_{0}$, so $a^{-1} \in A_{0}$. By definition, there is an element $b \in B$ such that $a^{-1} b \in G_{0}$. Thus there is an element $g \in G^{\prime}$ such that $a^{-1} b=(a, g)$. So $b=$ $g^{-1} a g$. $G=A B$ however so $g=a_{0} b_{0}$ for some $a_{0} \in A, b_{0} \in B$. Thus $b=$ $b_{0}^{-1} a_{0}^{-1} a a_{0} b_{0}=b_{0}^{-1} a b_{0}$. Conjugating by $b_{0}^{-1}$ we get $1 \neq a=b \in A \cap B$. Contradiction.

\section{REFERENCES}

1. N. Itô, Über das Produkt von zwei abelschen Gruppen, Math. Z. 62 (1955), 400-401. MR 17, 125.

2. P. M. Cohn, A remark on the general product of two infinite cyclic groups, Arch. Math. 7 (1956), 94-99. MR 18, 376.

3. N. F. Sesekin, The product of finitely connected abelian groups, Sibirsk. Mat. Ž. 9 (1968), 1427-1430=Siberian Math. J. 9 (1968), 1070-1072. MR 38 \#4559.

4. E. Schenkman, The general product of two finitely generated abelian groups, Proc. Amer. Math. Soc. 21 (1969), 202-204; Zentralblatt Math. 174 (1969), 308. MR 38 \#4565.

5. W. R. Scott, On a result of Schenkman on products of abelian groups, Notices Amer. Math. Soc. 16 (1969), 796. Abstract \#667-137.

6. B. Amberg and W. R. Scott, Products of abelian subgroups, Proc. Amer. Math. Soc. 26 (1970), 541-547. MR 42 \#1903.

7. L. E. Knop, Groups which are products of two abelian subgroups, Notices Amer. Math. Soc. 19 (1972), A-91. Abstract \#691-20-24.

8. B. Amberg, Groups which are the product of two abelian subgroups, Notices Amer. Math. Soc. 19 (1972), A-570. Abstract \#72T-A191.

Department of Mathematics, University of Utah, Salt Lake City, Utah 84112 